Published in final edited form as:

Biomed Eng (Singapore). 2017 August ; 29(4): . doi:10.4015/S1016237217500314.

\title{
SIMULATION ANALYSIS OF BLOOD FLOW IN ARTERIES OF THE HUMAN ARM
}

\author{
Wu Quanyu ${ }^{*} \neq$, Liu Xiaojie*, Pan Lingjiao*, Tao Weige ${ }^{*}$, and Qian Chunqi ${ }^{\dagger}$ \\ *Institute of Bioinformatics and Medical Engineering, School of Electrical and Information \\ Engineering, Jiangsu University of Technology, Changzhou 213001, Jiangsu, P. R. China \\ †Department of Radiology, Michigan State University, East Lansing, MI 48864, USA
}

\begin{abstract}
Arteries in the upper limb play important roles in the circulation system of the human body. In particular, the radial artery has received considerable attention in traditional Chinese medicine for thousands of years. Here, a 3D model for the arm arteries has been created uncomplicated, in a Chinese adult's left hand, from the magnetic resonance imaging data, using professional modeling software to restore the basic structure of the arm artery in human body, before being imported to Ansys software for simulation. Blood model has been only simulated, and using the blood density of constant parameter and viscosity using the Carreau fluid model, and using viscous-laminar model of Fluent to obtain the velocity profile, static pressure and shear stress in the brachial, interosseous, ulnar, radial and palmar arch arteries. In particular, the brachial and bifurcations have the high pressure and velocity profiles. The simulation results obtained here are also validated by those published in the literature and proved the ulnar artery prevails over the radial artery as a blood supplier to the vessels in the wrist and hand.
\end{abstract}

\section{Keywords}

Human arm artery; Blood flow; Velocity profile; Static pressure; Numerical simulation

\section{INTRODUCTION}

Computational analysis of blood flow in human has been extensively studied in the last decade. ${ }^{1,2}$ Due to the close relation between hemodynamics and the various ailments in the human body, ${ }^{3}$ Leng et al. ${ }^{4}$ and Saho and Onishi ${ }^{5}$ simulated hemodynamics in 3D angiography cerebral models. They studied intracranial atherosclerotic stenosis and cerebral aneurysms, respectively, but results of Saho and Onishi indicated that the cause of cerebral aneurysms remained unclear. The algorithm to incorporate both a Newtonian fluid physics model and a linear PC-MRI signal model was proposed by Rispoli et al. ${ }^{6}$ for carotid

\footnotetext{
${ }^{\ddagger}$ Corresponding author: Wu Quanyu, Institute of Bioinformatics and Medical Engineering, School of Electrical and Information Engineering, Jiangsu University of Technology, 1801 Zhongwu Avenue, Changzhou 213001, Jiangsu, P. R. China. Tel: (+86) 519-86953225; wuquanyu@jsut.edu.cn.

CONFLICTS OF INTEREST

There are no conflicts of interest to disclose.
} 
bifurcation, where regularized blood flow gained the best results. Gharahi et al. ${ }^{7}$ simulated human carotid artery bifurcation based on anatomy and volumetric blood flow rate measured with PC-MRI. They found the limitation of PC-MRI to be its non-precise measurement of blood velocity near artery walls, although CFD simulation gave better results. Potters et al ${ }^{8}$ also studied the carotid bifurcation and quantified wall shear stress near artery wall on 4D MRI. Recently, the correlation between thoracic aortic diseases and hemodynamics has received considerable attention; however, the underlying causes of these diseases have not been fully understood. Suito et al. ${ }^{9}$ analyzed the blood flow and geometrical characteristics in the thoracic aorta, using the ST-VMS method to solve fluid mechanics equations. Al-Rawi and Al-Jumaily ${ }^{10}$ studied abdominal aorta narrowing using computational fluid dynamics and found that arterial blockage in excess of $20 \%$ of the diameter significantly influences the pressure wave and reduces the systolic blood pressure at the right femoral artery. Nestola et al. ${ }^{11}$ performed simulation for abdominal aortic aneurysms, bringing attention to three-band decomposition methodology for analysis of moving domains. The importance of shunt angulations as a determinant of shear-stress-induced thrombosis was proposed by Celestin et al., ${ }^{12}$ who analyzed characteristics of blood flow from central aorta to pulmonary artery. Their results indicated that central aortic shunts of longer length and with angulations were easy to get clotted. Although there are a few studies on hemodynamics of coronary artery bypass grafts ${ }^{13}$ and pulmonary arterial blood flow, ${ }^{14}$ little attention was given to the simulation of small arteries, such as the radial artery and the dorsalis pedis artery. The method of radial artery point has been used by Chinese medicine for thousands of years, to obtain more information about human body and some diseases. In 2013, Watanabe et al. ${ }^{15}$ focussed on the arterial network of the arm; the model comprised 108 arterial segments with 63 main arteries and 44 perforator arteries, with lumen radii ranging from $0.24 \mathrm{~cm}$ axillary artery to $0.018 \mathrm{~cm}$ perforator arteries, which was a complicated project. Furthermore, their results of pressure wave and flow rate were in agreement with patients' records already published in the literature. In 2016, Choudhari and Panse ${ }^{16}$ simulated the flow in artery in human arm to study the aortic pulse wave propagation; they used finite element model for ascending aorta, carotid, brachial, interosseous, ulnar and radial artery, but not the palmar arch artery. Furthermore, they simulated using COMSOL multi-physics and obtained the static pressure, velocity profile and wall shear stresses, simulation results of which were supported by comparing the standard published results. Kim et al. simulated the arterial tree in human upper extremity; their model included radial, ulnar, deep and superficial palmar arch arteries, but not the interosseous artery. In addition, they created a three-dimensional model from PC-MRI data and analyzed the velocity, pressure and shear stress images from the artery model and proved physiological blood flows near the branches of radial and deep palmar arch arteries and ulnar and superficial palmar arch arteries.

This paper aims to simulate the arterial tree in the entire human arm and investigates some hemodynamic parameters as blood propagates from the brachial artery toward the palmar arch arteries. Our model includes brachial, radial, interosseous, ulnar and palmar arch arteries, all of which were studied little previously. ${ }^{15-17}$ We analyze the velocity, static pressure, shear stress and compare with the phenomena derived from parameters measured by noninvasive measurement devices, such as blood pressure gage, the applanation tonometry-based automated vascular testing device ${ }^{18-20}$ and the heart rate monitor. Based on 
our simulating characteristic results, these devices can not only monitor the anthropometric parameters obviously, but also help to analyze the hemodynamic parameters. The materials and methods are elaborated in detail in Sec. 2, and the results are given in Sec. 3. The discussion is presented in Sec. 4, and the concluding remarks are described in Sec. 5.

\section{MATERIALS AND METHODS \\ Geometry and Physical Parameters}

Here we mainly consider the arteries of the human arm artery. This model was constructed from the MRI data set of a male subject and not based on using image commercial modeling tools. We used professional modeling software to construct the model by manual. Figure 1 shows the synthetic model of artery and blood. The model mainly included two parts: one is the arterial model and the other is the blood model in matching.

The arterial model has a length of $80.093 \mathrm{~mm}$ along the $x$-axis, $421.31 \mathrm{~mm}$ along the $y$-axis and $4.9 \mathrm{~mm}$ along with $z$-axis, with a wall thickness of $0.3 \mathrm{~mm}$. Then the blood model has a length of $79.538 \mathrm{~mm}$ along the $x$-axis, $421.22 \mathrm{~mm}$ along the $y$-axis and $4.3 \mathrm{~mm}$ along the $z$ axis in matching. Because the stringent anatomical and physiological criteria are complicated, ${ }^{15}$ here we select the main parts of the arm arteries, which include the brachial, interosseous, ulnar, radial and palmar arch arteries in detail. The blood flows into the inlet on the right and to six outs on the left; here the inlet represents the main brachial; out1 represents the radial collateral; out 2 and out 4 represent recurrent, anterior, posterior and common interosseous area; out 3 represents ulnar out area; out5 and out 6 represent deep, superficial and common palmar ach artery. Table 1 shows vessel diameters.

Here, we model the blood density of $1060 \mathrm{~kg} / \mathrm{m}^{3}$ and viscosity using the Carreau fluids model. Table 2 shows parameters of the Carreau model in Ansys Fluent.

Human heart ejection is cyclically time-varying. Thus the velocity at the inlet of brachial artery is not set to be a constant, but instead with a time-varying periodic profile. Here we used cardiac cycle model and defined the UDF in Fluent. The sine wave in the systolic phase has a peak velocity of $1.2 \mathrm{~m} / \mathrm{s}$ and a minimum velocity of $0.1 \mathrm{~m} / \mathrm{s}$, assuming a heartbeat rate of $75 / \mathrm{min}$; the duration of each period is $0.8 \mathrm{~s}$. Figure 2 shows the flow velocity waveform at the inlet boundary. The six outs pressures are set to be zero with natural outflow, and we do not use the anatomical and physiological criteria to control the blood flow rate at each outlet by means of a suitable terminal resistance.

In contrast, in order to describe the inlet profile more clearly, a UDF function is also given below in Eq. (1). $t$ is the simulation time:

$$
v= \begin{cases}1.2 * \sin (4 * \pi *(t+1.6)) & t \leq 2.18 \\ =0.1 & t>2.18\end{cases}
$$




\section{Meshes Parameters}

Figure 3 shows the mesh part model of blood geometry. The blood mechanics meshes are made of four-noded tetrahedral elements, and the algorithm is patch conforming method. Patch conforming meshing is a meshing technique in which all faces and their boundaries within a very small tolerance are respected for a given part; mesh-based disfeaturing is used to overcome difficulties with small features and dirty geometry. Patch conforming meshing is invariant to loads and boundary conditions, namely, selections, results or any scoped object. We can implement patch conforming meshing using settings related to any parameters. In addition, the patch conforming tetra mesh method is a Delaunay tetra mesh with an advancing front point insertion technique used for mesh refinement.

We used advanced size function which was set on proximity and curvature. The relevance center and span angle center was set to fine; at the same time, the smoothing function was set to high and the transition function was set to slow. Finally, we got the statistics of the meshes: the number of node was 929,483, and elements were 604,087 in the blood body. Table 3 provides the details of set parameters in Ansys.

\section{Simulation Methods}

In this study, the simulation is performed with Ansys Fluent software (ANSYS Co., USA). Before starting a fluid simulation, we always pay attention to the governing equations of the underlying physics. In this case, the small arterial blood flow is usually a laminar and threedimensional flow, in which blood is considered to be an incompressible fluid. So the most fundamental governing equations is the Navier-Stokes equation, ${ }^{9}$ in which blood can be regarded as an incompressible fluid and the rate of density change is zero. Equation (2) expresses the Navier-Stokes equation:

$$
\left\{\begin{array}{l}
\frac{\partial \mathbf{u}}{\partial t}-\frac{\mu}{\rho} v \nabla^{2} \mathbf{u}+\mathbf{u} \nabla \bullet \mathbf{u}+\frac{1}{\rho} \nabla p=\mathbf{f} \\
\nabla \bullet \mathbf{u}=0
\end{array}\right.
$$

where $\mathbf{u}$ is the blood velocity, $p$ the pressure, $\rho$ the density, $\mu$ the viscosity, $v$ the coefficient of kinematic viscosity, $t$ the time and $\mathbf{f}$ the force.

According to Eq. (2), we find that the parameter of viscosity of $\mu$ is not a constant. Here, we model the blood viscosity using the Bird-Carreau law in Eq. (3):

$$
\eta=\eta_{\infty}+\left(\eta_{0}-\eta_{\infty}\right)\left(1+\lambda^{2} \dot{\gamma}^{2}\right)^{n-1 / 2}
$$

where $\eta$ is the effective viscosity, $\eta_{\infty}$ the infinite shear rate viscosity, $\eta_{0}$ the zero shear rate viscosity, $\lambda$ the natural time, $n$ the power law index; they are the material coefficients. 


\section{RESULTS}

We used double precision of the transient simulation in Fluent and pressure velocity coupling in PISO scheme; the residual monitor check convergence was set to 0.0001 . The entire blood calculation was running from static fluid, and the time stepping method was set to fixed, time step size was $0.1 \mathrm{~s}$, the number of time steps was 8 , the max iterations were 200 per time step. Finally, we used Ansys CFD-Post to deal with the calculation results starting from $0 \mathrm{~s}$ to $0.8 \mathrm{~s}$ of the entire range and obtained the velocity vector profile, static pressure and shear stress in the form of slices, vector, contours, iso-surfaces, etc. We selected four time types in analysis; they were T1 (0.1 s), T2 (0.2 s), T6 (0.6 s) and T8 (0.8 s), which especially represented different blood flow velocities in the inlet area. T1 was the highest velocity $1.2 \mathrm{~m} / \mathrm{s}$ at the peak systolic, T2 was near $0.2 \mathrm{~m} / \mathrm{s}$ at the late systolic and T6 and $\mathrm{T} 8$ were $0.1 \mathrm{~m} / \mathrm{s}$ for a period time at the late diastolic.

\section{Velocity Profile in Blood Model}

Figure 4 shows velocity lines at different times. First, Out1 represents the radial collateral and has the highest velocity in T1, which is near $5.8 \mathrm{~m} / \mathrm{s}$, and it is 4.8 times larger than 1.2 $\mathrm{m} / \mathrm{s}$ of the inlet velocity. But the common radial has a low velocity and near $0.1 \mathrm{~m} / \mathrm{s}$. Secondly, it has high velocity in the main bifurcation and it has crossed $2.8 \mathrm{~m} / \mathrm{s}$, and the second bifurcation has high velocity. On the other hand, we can find that Out2 and Out3 have the same velocity based on Fig. 4. Here the brachial artery keeps low velocity, but the ulnar and interosseous arteries have high velocity compare to radial artery in the laminar fluid model and is near $2.8 \mathrm{~m} / \mathrm{s}$. Finally, the remote radial indices artery has a high speed and near $2.85 \mathrm{~m} / \mathrm{s}$, which appears to be a strange phenomenon. However, the palmar arch artery has tiny velocity on the whole. In T2, the outs of the entire blood velocity is decreasing with the inlet velocity and keeps $1.5 \mathrm{~m} / \mathrm{s}$ in the vicinity and keeps steady in T8.

\section{Static Pressure}

Figure 5 shows maximum and minimum static pressures of blood developed during different times during the cardiac cycle. In $\mathrm{T} 1$ of the peak systolic, the inlet velocity has a maximum value, which leads to the highest static pressure in inlet and high pressure in the entire blood model. In particular, the inlet and first bifurcation have maximum pressure, but the out 1 has minimum value in comparison to inlet. Our results of the radial artery are mostly the same as in previous reports, ${ }^{17}$ but with a few difference for the ulnar artery, because the blood pressure in our model is low when compared with theirs. At the same time, we can find that many bifurcations have higher pressure. However, the outs of arteries keep the low static pressure, which is in agreement with the printed paper. ${ }^{15}$

\section{Shear Stress}

Figure 6 shows three time sections of shear stress in the blood model during the cardiac cycle. In T1 and T2, the Out1 appears shear stress obviously, and there is shear stress in ulnar bifurcation artery as well, here the shear stress appears obviously. The bifurcations of Out 2 and Out 3 have shear tress, but with lower magnitude. On the other hand, the palmar arch has no shear stress, and our results are the same as in printed paper. ${ }^{17}$ But shear stress is in the second bifurcation of blood model in $\mathrm{T} 2$. 


\section{DISCUSSIONS}

People place more and more emphasis on health in the 21st century, but the cardiovascular diseases were the top 10 leading causes of death in USA by the report of the Centers for Disease Control and Prevention. So we paid attention to study the hemodynamic simulation, in addition, combined with noninvasive electronic instruments to evaluating the physical condition. The human arm artery is a special position, and the clinical staff tests the blood pressure from here for many years. At the same time, the radial artery has received considerable attention in traditional Chinese medicine for thousands of years.

Here we selected the main parts of the arm arteries, which include the brachial, interosseous, ulnar, radial and palmar arch arteries. Choudhari and Panse ${ }^{16}$ selected the ascending aorta, carotid, brachial, interosseous, ulnar and radial arteries; then their model did not include palmar arch artery. Kim et al. ${ }^{17}$ simulated the model including radial, ulnar, deep and superficial palmar arch arteries, but not the interosseous artery. Watanabe et al. ${ }^{15}$ constructed the complicated model topology, and their model comprised 108 arterial segments, with 64 main arteries and 44 perforator arteries, and so therefore need a long simulating time. The static pressure and velocity profiles were shown at different times in three papers and appeared in the brachial and bifurcations obviously. Based on our results, Figs. 4 and 5 proved the previous reports, respectively. The heart ejection time was in $\mathrm{T} 1$ of the peak systolic state, the arteries blood got high velocity, static pressure and shear stress in brachial, radial and main bifurcations. However, the microarteries were influenced unobviously based on our simulated results. In particular, Fig. 5 shows the shear stress. Therefore, this phenomenon explains why the small diameters and distal vessels receive less influence.

Figure 4 shows the velocity profiles in the geometry model, comparison to the printed paper, ${ }^{15}$ in their simulation model; the mean blood flow through the ulnar artery results $\left(0.7069 \mathrm{~cm}^{3} / \mathrm{s}\right)$ is higher than the radial artery of the mean blood $\left(0.6627 \mathrm{~cm}^{3} / \mathrm{s}\right)$. Based on our simulation results, the average blood velocity of the ulnar artery is higher than the common radial artery, which also proves that the ulnar artery prevails over the radial artery as a blood supplier to the vessels in the wrist and hand. In addition, the phenomena were most common situations encountered in the clinical diagnosis process. According to our simulation results, the artery of remote radial indices appeared high velocity profile, which was caused by the return flow and elastic artery. Before we always studied the radial pulse waveform by the applanation tonometry-based automated vascular testing device, ${ }^{18}$ the radial pulse waveform existed the reflected wave. ${ }^{21}$ Therefore, these findings proved the simulation results.

Figures 5 and 6 show the pressure and shear stress, respectively. The results obtained using the Fluid simulations were found to be different in artery positions. The result of the pressure change represented the vessel dispensability and blood flow distribution, which influenced the wall of the deformable artery. The shear stress played an important role in the natural history of atherosclerosis and aneurysm. These arterial diseases in the arm arteries are less common than those in aorta and cerebral arteries. However, the shear stress of the experimental and numerical investigations related to the arm artery has been rarely 
performed. Based on our results, the shear stress was obtained in the bifurcations and proximal ulnar artery obviously.

Now we tried to study the hemodynamics about the human arm arteries. First, we only simulated the blood model by the Fluent software and grasped knowledge of hemodynamics and constructed the artery model. Secondly, we loaded the pressure into the artery model by transient structure software and simulated the artery reformation, equivalent stress, shear stress and equivalent total strain. Finally, combined with Ansys software and electronic instruments furthermore developed the Ansys of secondary software to assess the health status of the subjects.

\section{CONCLUSIONS}

This paper has provided the finite element model for arteries using the professional modeling software, given the simulation results of the arteries in the human arm. Previously, most researchers have not studied such small arteries. Here, we consider the arterial network including the brachial, interosseous, ulnar, radial and palmar arch arteries. The velocity profile of Out1 is 4.8 times larger than $1.2 \mathrm{~m} / \mathrm{s}$ of the inlet velocity. The shear stress mainly appears in the bifurcations of the artery model, whereas the palmar arch arteries have almost no shear stress. The maximum pressure is obtained when the inlet velocity has a maximum value, especially in the radial artery. Therefore, our results proved that we got the pulse waveform easily at the radial artery point by noninvasive instruments. In contrast, based on our results, we could check the pulse wave in the radial point by many noninvasive instruments. In the future, in order to pave the way for clinical application, we will take further steps to seek improvement in arm artery modeling, for the simulation of the bidirectional fluid structure coupling in the human arm arteries.

\section{Acknowledgments}

This research is funded by the Changzhou Social Development Foundation (Grant No. CE20165021) and Jiangsu Science and Technology Project (Grant No. 2016030-20).

\section{References}

1. Alastruey J, Xiao N, Fok H, Schaeffter T, Figueroa CA. On the impact of modelling assumptions in multi-scale, subject-specific models of aortic haemodynamics. J R Soc Interf. 2016; 13:1-17.

2. Mittal R, Seo JH, Vedula V, Choi YJ, Liu H, Huang HH, Jain S, Younes L, Abraham T, George RT. Computational modeling of cardiac hemodynamics: Current status and future outlook. J Comput Phys. 2016; 305:1065-1082.

3. Ramachandra AB, Kahn AM, Marsden AL. Patient-specific simulations reveal significant differences in mechanical stimuli in venous and arterial coronary grafts. J Cardiovasc Transl Res. 2016; 9:279-290. [PubMed: 27447176]

4. Leng X, Scalzo F, Fong AK, Johnson M, Ip HL, Soo Y, Leung T, Liu L, Feldmann E, Wong KS, Liebeskind DS. Computational fluid dynamics of computed tomography angiography to detect the hemodynamic impact of intracranial atherosclerotic stenosis. Neurovasc Imaging. 2015; 1:1-7.

5. Saho T, Onishi H. Quantitative comparison of hemodynamics in simulated and 3d angiography models of cerebral aneurysms by use of computational fluid dynamics. Radiol Phys Technol. 2015; 8:258-265. [PubMed: 25911446] 
6. Rispoli VC, Nielsen JF, Nayak KS, Carvalho JL. Computational fluid dynamics simulations of blood flow regularized by $3 \mathrm{~d}$ phase contrast mri. Biomed Eng Online. 2015; 14:110. [PubMed: 26611470]

7. Gharahi H, Zambrano BA, Zhu DC, DeMarco JK, Baek S. Computational fluid dynamic simulation of human carotid artery bifurcation based on anatomy and volumetric blood flow rate measured with magnetic resonance imaging. Int J Adv Eng Sci Appl Math. 2016; 8:40-60. [PubMed: 27546999]

8. Potters WV, Cibis M, Marquering HA, vanBavel E, Gijsen F, Wentzel JJ, Nederveen AJ. 4d mribased wall shear stress quantification in the carotid bifurcation: A validation study in volunteers using computational fluid dynamics. J Cardiovasc Magn Reson. 2014; 16:P162.

9. Suito H, Takizawa K, Huynh VQH, Sze D, Ueda T. Fsi analysis of the blood flow and geometrical characteristics in the thoracic aorta. Comput Mech. 2014; 54:1035-1045.

10. Al-Rawi M, Al-Jumaily AM. Assessing abdominal aorta narrowing using computational fluid dynamics. Med Biol Eng Comput. 2016; 54:843-853. [PubMed: 26319006]

11. Nestola MGC, Gizzi A, Cherubini C, Filippi S. Three-band decomposition analysis in multiscale fsi models of abdominal aortic aneurysms. Int J Mod Phys C. 2016; 27:1650017.

12. Celestin C, Guillot M, Ross-Ascuitto N, Ascuitto R. Computational fluid dynamics characterization of blood flow in central aorta to pulmonary artery connections: Importance of shunt angulation as a determinant of shear stress-induced thrombosis. Pediatr Cardiol. 2015; 36:600-615. [PubMed: 25404555]

13. Ballarin F, Faggiano E, Ippolito S, Manzoni A, Quarteroni A, Rozza G, Scrofani R. Fast simulations of patient-specific haemodynamics of coronary artery bypass grafts based on a PODGalerkin method and a vascular shape parametrization. J Comput Phys. 2016; 315:609-628.

14. Qureshi MU, Vaughan GD, Sainsbury C, Johnson M, Peskin CS, Olufsen MS, Hill NA. Numerical simulation of blood flow and pressure drop in the pulmonary arterial and venous circulation. Biomech Model Mechanobiol. 2014; 13:1137-1154. [PubMed: 24610385]

15. Watanabe SM, Blanco PJ, Feijóo RA. Mathematical model of blood flow in an anatomically detailed arterial network of the arm. ESAIM: Math Model Numer Anal. 2013; 47:961-985.

16. Choudhari P, Panse MS. Finite element modeling and simulation of arteries in the human arm to study the aortic pulse wave propagation. Proc Comp Sci. 2016; 93:721-727.

17. Kim K, Kim JU, Beak HM, Kim SK. Modeling of the artery tree in the human upper extremity and numerical simulation of blood flow in the artery tree. Trans Kor Soc Mech Engrs B. 2016; 40:221226.

18. Wu Q-Y, Ma Z-C, Sun Y-N. Noninvasive power spectrum analysis of radial pressure waveform for assessment of vascular system. J Mech Med Biol. 2012; 12:1250021.

19. Hashimoto J, Ito S. Pulse pressure amplification, arterial stiffness, and peripheral wave reflection determine pulsatile flow waveform of the femoral artery. Hypertension. 2010; 56:926-933. [PubMed: 20876451]

20. Avolio AP, Van Bortel LM, Boutouyrie P, Cockcroft JR, McEniery CM, Protogerou AD, Roman MJ, Safar ME, Segers P, Smulyan H. Role of pulse pressure amplification in arterial hypertension: Experts' opinion and review of the data. Hypertension. 2009; 54:375-383. [PubMed: 19564542]

21. Zhang YL, Zheng YY, Ma ZC, Sun YN. Radial pulse transit time is an index of arterial stiffness. Hypertens Res. 2011; 34:884-887. [PubMed: 21593741] 


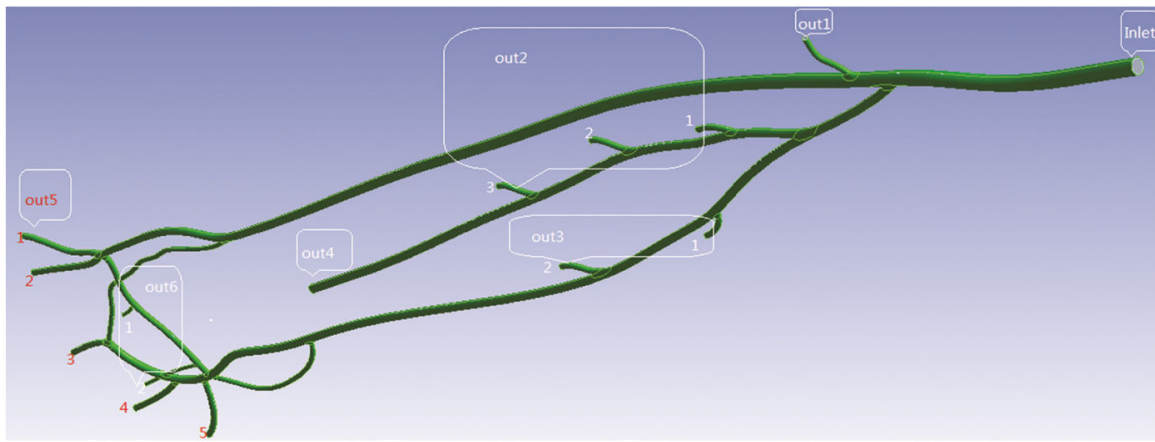

Fig. 1.

Artery model with blood in the human arm. 


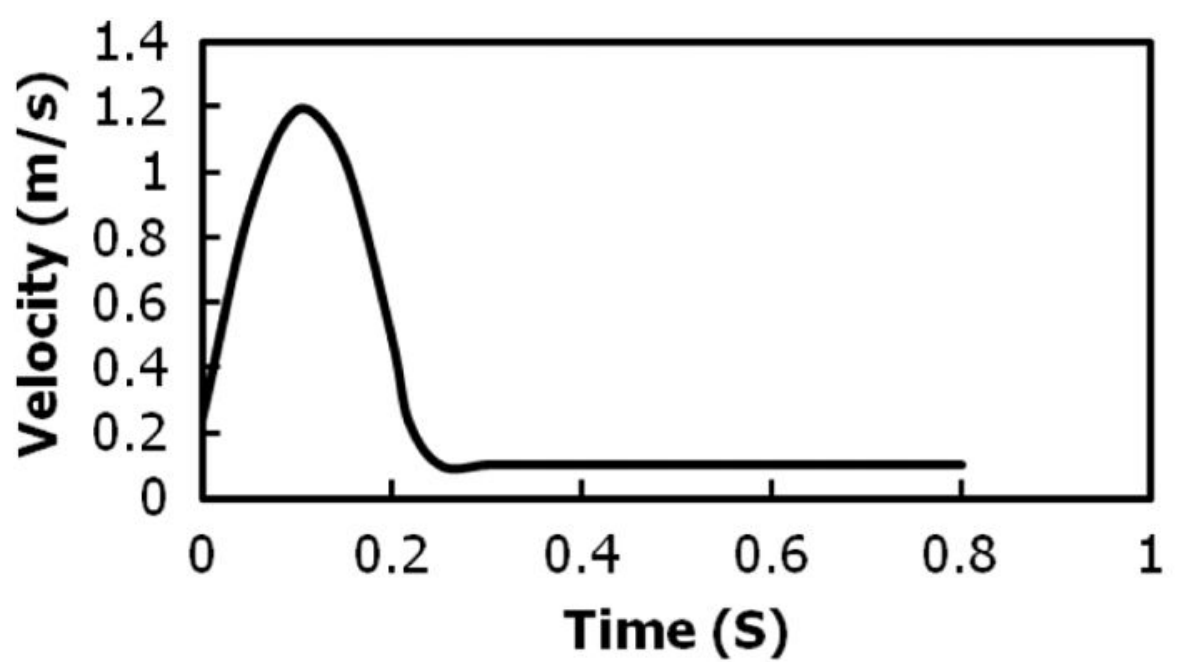

Fig. 2.

Flow velocity waveform in the inlet. 


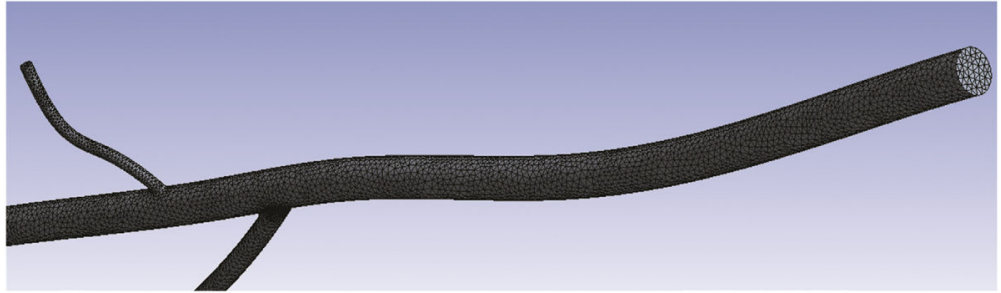

Fig. 3.

Mesh model of the blood geometry. 

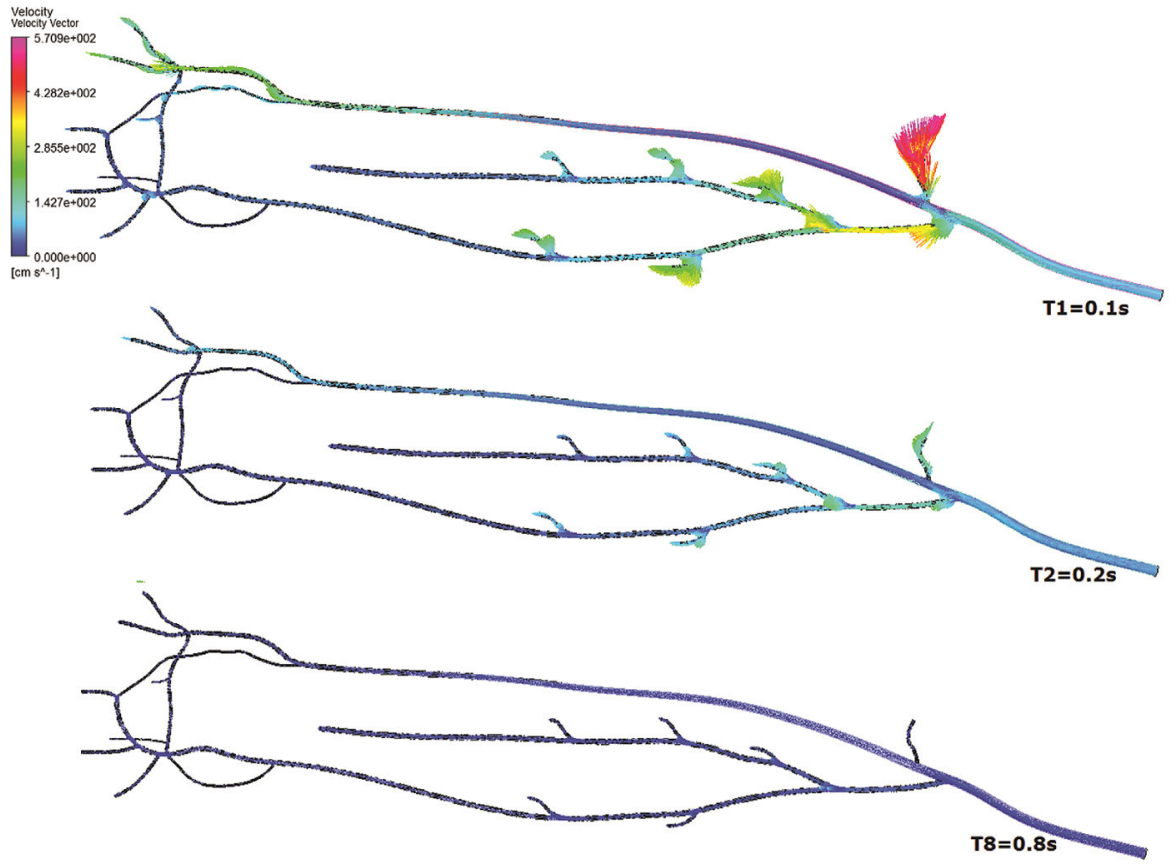

Fig. 4.

Velocity profile for blood model. 

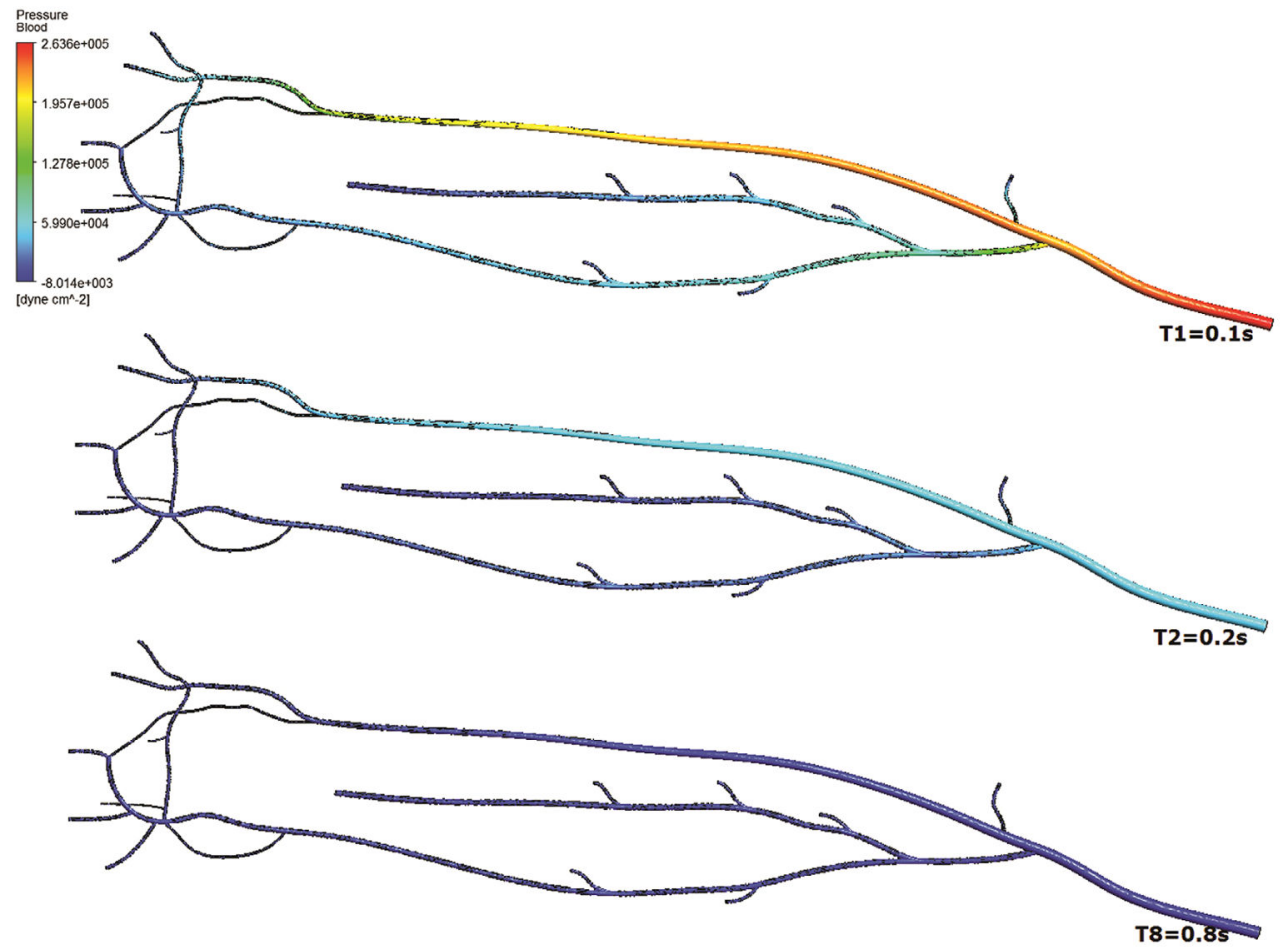

Fig. 5.

Static pressure plot for blood model. 

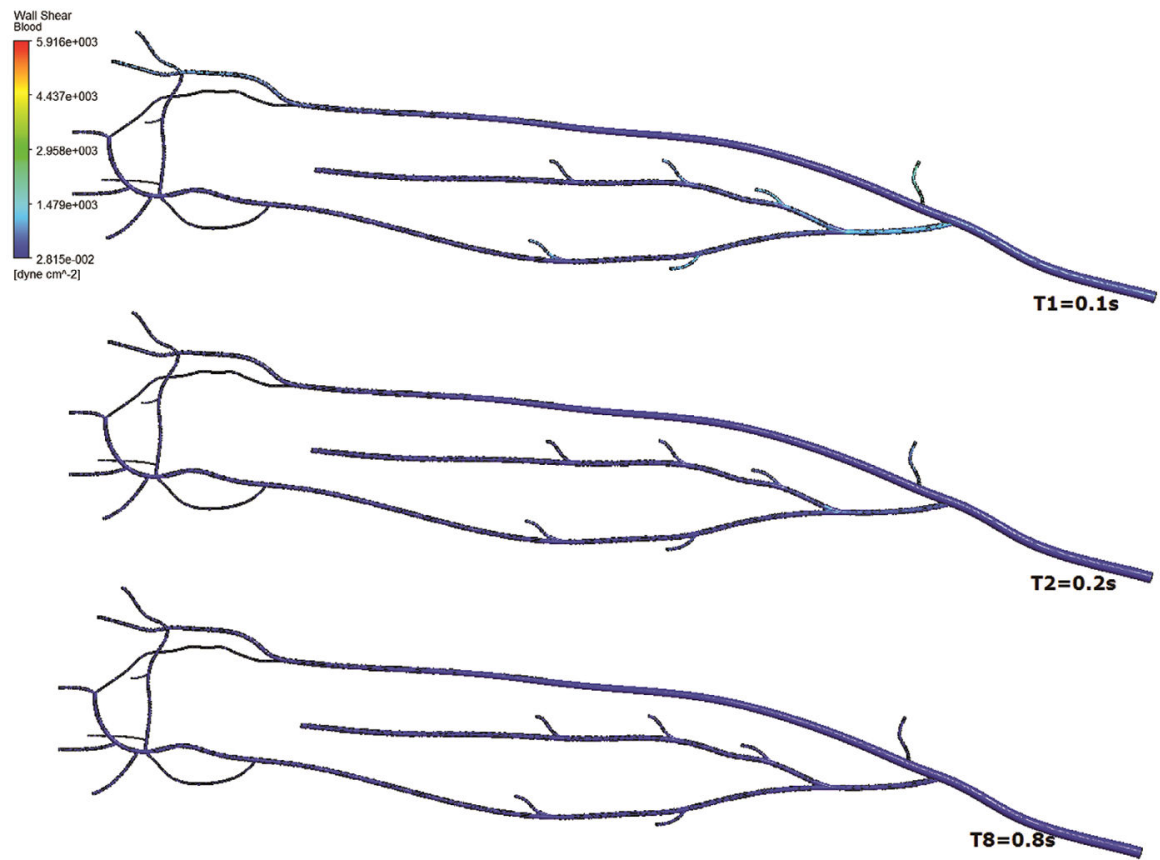

Fig. 6.

Shear stress plot for blood model. 

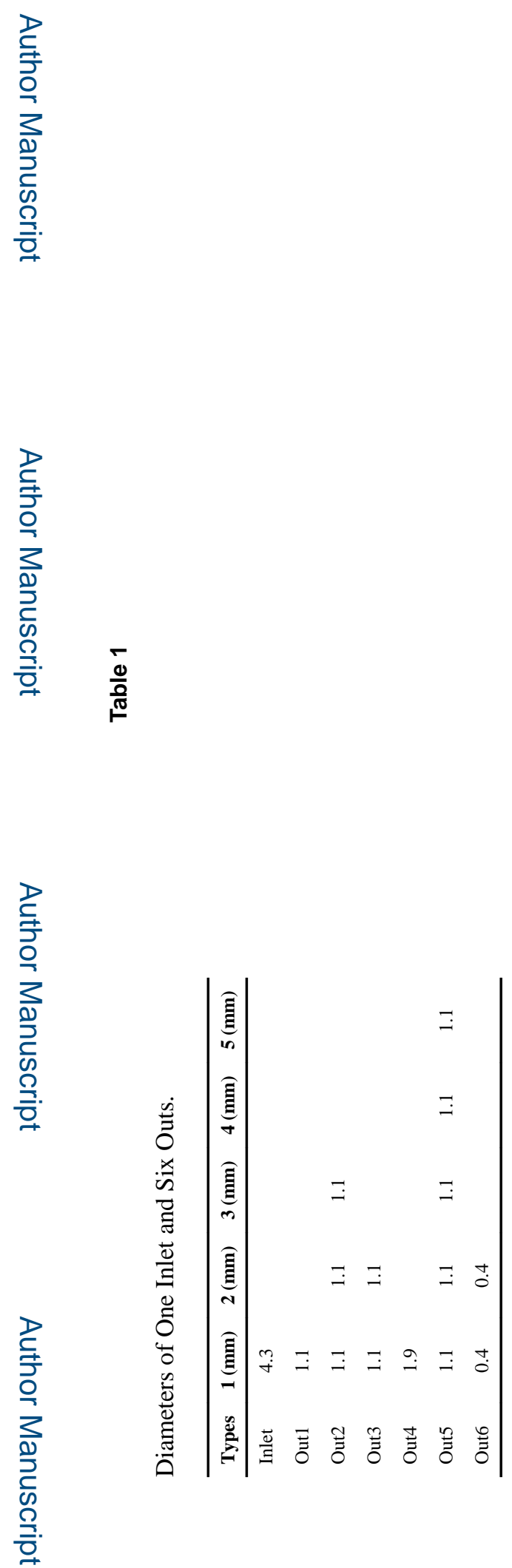

Biomed Eng (Singapore). Author manuscript; available in PMC 2018 February 01. 


\section{Table 2}

Details of Carreau Model Parameters in Fluent.

\begin{tabular}{ll}
\hline Coefficients & Input Value \\
\hline Time constant, lambda (s) & 3.313 \\
Power-law index, $n$ & 0.3568 \\
Zero shear viscosity $(\mathrm{kg} / \mathrm{m} \mathrm{s})$ & 0.056 \\
Infinite shear viscosity $(\mathrm{kg} / \mathrm{m} \mathrm{s})$ & 0.0035 \\
\hline
\end{tabular}



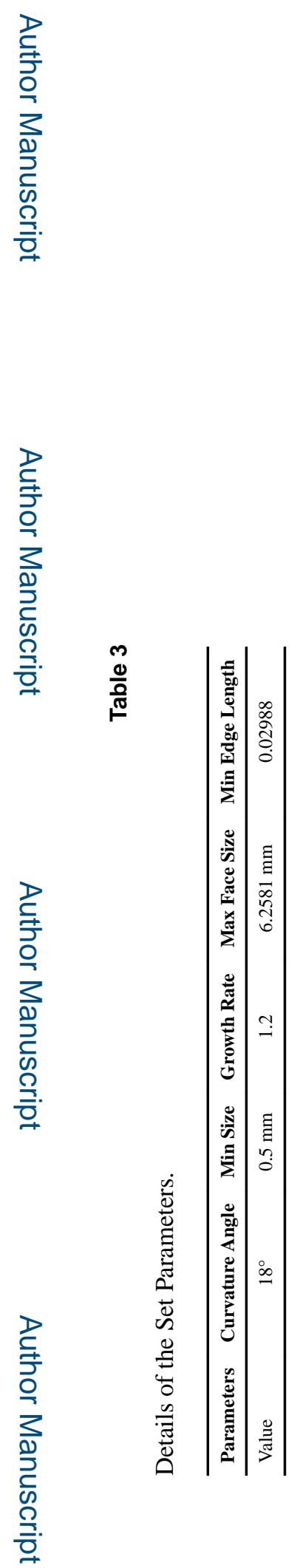

Biomed Eng (Singapore). Author manuscript; available in PMC 2018 February 01. 